\title{
BMJ Open Outcomes and care priorities for older people living with frailty and advanced chronic kidney disease: a multiprofessional scoping review protocol
}

\author{
Helen Hurst (D) , ${ }^{1}$ Elizabeth Jones, ${ }^{2}$ Paula Ormandy, ${ }^{3}$ Alison Brettle, ${ }^{4}$ \\ Andrew Christopher Nixon (D) , ${ }^{5,6}$ Hannah Young, ${ }^{7,8}$ Andrew Mooney, ${ }^{9}$ \\ Anna Winterbottom, ${ }^{10}$ Hilary Bekker, ${ }^{11}$ Edwina Brown, ${ }^{12}$ Fliss EM Murtagh, ${ }^{13}$ \\ Maria Da Silva-Gane, ${ }^{14,14}$ David Coyle, ${ }^{15}$ Robert Finnigan ${ }^{15}$
}

To cite: Hurst $\mathrm{H}$, Jones $\mathrm{E}$, Ormandy P, et al. Outcomes and care priorities for older people living with frailty and advanced chronic kidney disease: a multiprofessional scoping review protocol. BMJ Open 2021;11:e040715. doi:10.1136/ bmjopen-2020-040715

- Prepublication history and additional material is published online only. To view please visit the journal online (http://dx.doi. org/10.1136/bmjopen-2020040715).

Received 20 May 2020

Revised 22 December 2020

Accepted 30 December 2020

D Check for updates

(C) Author(s) (or their employer(s)) 2021. Re-use permitted under CC BY-NC. No commercial re-use. See rights and permissions. Published by BMJ.

For numbered affiliations see end of article.

Correspondence to

Dr Helen Hurst;

helen.hurst@mft.nhs.uk

\section{ABSTRACT}

Introduction Reported outcomes for older people with advanced chronic kidney disease (CKD) often focus on survival and mortality and little attention is paid to symptom burden and health-related quality of life. Recognising frailty and providing interventions that may improve outcomes have been studied in the general population with a growing research interest within CKD.

Methods and analysis A scoping review will be undertaken following a recommended process to understand relevant research and priorities for older people living with frailty and advanced CKD. Databases will be searched and following a systematic process by a core team, a final list of included studies will be analysed. Focus groups will then be conducted with older people with advanced CKD to incorporate stakeholder views.

Ethics and dissemination Our scoping review will use robust methodology to identify relevant literature focused on outcomes and care priorities for older people with advanced CKD. Ethical approval will be sought to conduct the focus groups. The result of this review will be disseminated through patient networks and national conferences. The interdisciplinary team collaborating plan to continue work in this area to improve the care and management of older people with advanced CKD.

\section{BACKGROUND}

The ageing population is a major concern for both health and social care across the UK. The construct of frailty, which recognises the multidimensional patterns of decline in older people, has been used to categorise vulnerable older adults at greatest risk of adverse health outcomes. ${ }^{2}$ This increased risk has driven the need to identify frailty and associated geriatric syndromes among older people and offer a personalised management plan that aims to improve outcomes. ${ }^{2-4}$ Frailty arises from a combination of reduced resilience and increased vulnerability associated with ageing. The British Geriatric Society

\section{Strengths and limitations of this study}

- This is the first review to focus on outcome priorities for older people living with frailty and advanced chronic kidney disease (CKD).

- The research group has a breadth of expertise, including patient representatives and interdisciplinary members.

- A rigorous process will be followed to identify and select studies, but these will not be subject to an in-depth assessment of quality.

- The review will include a comprehensive range of peer-reviewed and grey literature but limited to English language papers.

- Older people living with frailty and advanced CKD and carers will share their perspectives on the findings and integrated into the final report.

defines frailty as 'a distinctive health state related to the ageing process in which multiple body systems gradually lose their in-built reserves diminishing the ability to carry out important practical and social activities. ${ }^{5}$ There are two principal conceptual models of frailty, specifically the deficit accumulation model of frailty (operationalised as the Frailty Index) and the physical model of frailty (often referred to as the Frailty Phenotype). ${ }^{367}$ Although differing in their underlying theories, both predict increased vulnerability to adverse outcomes. Since their publication, several screening tools have been derived that aim to offer a more clinically practical approach to frailty identification.

Frailty is not just a concern for geriatric medicine; studies within specialty medicine, including nephrology, have demonstrated the association between frailty and health outcomes in their respective patient populations. ${ }^{9}$ Reports of outcomes relevant to 
older people living with frailty in the general population cannot be generalised to those with chronic kidney disease (CKD) as there are notable differences between those who are frail with CKD and those who are frail with normal kidney function. ${ }^{210}$ Differences include increased falls, hospitalisation, lower health-related quality of life (HRQoL), increased symptom burden and mortality rate. ${ }^{10-21}$ Furthermore, studies that have investigated frailty in CKD populations not only report a higher prevalence of frailty but also frequently report frailty in younger age groups, potentially reflecting alternative drivers of the frailty syndrome in CKD. Importantly, it is not clear which outcomes are most important for older people living with frailty and advanced CKD.

Studies that focus on older people with advanced CKD typically focus on morbidity and mortality. ${ }^{22}$ These outcomes are unarguably relevant, although symptomburden, HRQoL and other patient-reported outcomes are also important. For example, when choosing between treatment options, patient decision-making is informed by not only survival outcomes but also lifestyle, HRQoL and preferences for end-of-life care. ${ }^{23}{ }^{24}$ Outcomes that are important for frail adults in the general population have been reported ${ }^{25}$ but unlikely to be the same as for frail adults with advanced CKD, and may not be relevant given the complexities of renal replacement therapy (RRT). Equally, outcomes important for patients with CKD in general are unlikely to be the same as for frail adults with the same condition. Although the Standardised Outcomes in Nephrology (SONG) initiative is working towards establishing core outcomes in $\mathrm{CKD}^{22}$ and the James Lind Alliance ${ }^{26}$ has recommended research priorities for patients living with frailty, to our knowledge, no group has yet looked at both groups in conjunction.

The comprehensive geriatric assessment (CGA) is a model of healthcare based on comprehensive multidisciplinary assessment taking account of medical, psychological, functional, social and environmental problems. The CGA is the recognised gold standard of care for older people living with frailty admitted to secondary $\operatorname{care}^{227}$ and has also been recommended in primary care settings. ${ }^{27} \mathrm{~A}$ recent systematic review identified a need for further research evaluating the role of a geriatric assessment in the advanced CKD population. ${ }^{28}$ This is also supported by the European Renal Best Practice Working Group emphasising the need 'to identify those who would benefit from more in-depth geriatric assessment and rehabilitation' and recommending further research in this area. ${ }^{29}$ A recent umbrella systematic review of the CGA indicated that key outcomes reported within existing reviews of the CGA in older adults without CKD were death, disability and institutionalisation, reduced length of stay and readmission, while patient-reported outcomes (PROMs) and HRQoL measures were fewer and reported less frequently. ${ }^{30}$ The focus of the CGA is to encompass a holistic approach to improve outcomes for people living with frailty relating to social and functional abilities and these potential gaps on important outcomes for older people living with frailty and advanced CKD.

This scoping review will identify and summarise existing evidence relating to relevant research and priorities for older people living with frailty and advanced CKD. The proposed investigation is part of a UK-wide collaborative working group of interprofessional experts, including clinicians, nurses, allied health professionals, health psychologists, decision scientists and applied health researchers. Research has highlighted that the experience of older people living with frailty and CKD is often not 'heard' by their healthcare professionals, emphasising the need to also understand the perspectives of people living with frailty and CKD, and their carers. ${ }^{31}$ The collaborative working group aims to identify research priorities and inform the design of improved models of care for older patients with advanced CKD.

This project will be conducted in accordance with the study protocol and the ethical principles outlined by Good Clinical Practice (GCP) and the Declaration of Helsinki.

\section{Aim}

The aim of this scoping review is to identify and summarise existing evidence relating to relevant research and priorities for older people living with frailty and advanced CKD (stages 4-5). Following preliminary discussion within the multiprofessional review team and a stakeholder group, comprising older people living with advanced CKD and their carers and family members, we identified four broad topics for further exploration within this review:

- Frailty identification and assessment methodology.

- Quantitative and qualitative outcomes reported for older people living with frailty and advanced CKD.

- Studied interventions that aim to improve outcomes for older people living with frailty and advanced CKD, including but not limited to survival, HRQoL, functional-ability, self-care, decision-making and cognition.

- How care priorities for older people living with frailty and advanced CKD can be better understood.

\section{METHODS}

The scoping review strategy follows a five-staged framework described by Arksey and O'Malley in 2005, which is outlined below under the headings that describe the process. The PRISMA-ScR guidelines for scoping reviews will also be used to guide the methods and ensure a systematic approach is followed. On completion of this review, they will be included as a checklist and appendix. ${ }^{32}$ The broad nature of scoping reviews requires a focus on summarising the breadth of evidence leading to a clearly articulated scope of inquiry. ${ }^{33}$ The questions identified below emphasise the importance of clarity in the concept, the population and outcomes of interest in order to develop an effective search strategy. ${ }^{33}$ 
Members of the research team form the collaborative group, although a core group will act as the review team including an expert in scoping review methodology.

\section{Stage 1: identifying the research question}

The broad research questions used in the initial scoping process include:

- What outcomes have been reported in older people living with frailty and advanced CKD?

- What interventions have been reported and how might they enhance both care and outcomes specifically for older people with advanced CKD?

- Is frailty formally identified in this population and if so what methods are used to identify frailty?

- What outcomes matter most to older people (living with frailty and advanced CKD), their relatives and carers?

In keeping with the recommended iterative methodology of scoping reviews, the research questions may be refined further as the review progresses.

\section{Inclusion and exclusion criteria}

The initial inclusion and exclusion criteria have been written to focus the search strategy but may need to be altered at later stages of the review process.

\section{Inclusion criteria}

- Population: Older people (aged $\geq 65$ years) with advanced CKD (stages 4 and 5), who may be receiving RRT, conservative care or have received a renal transplant, and carers/families of these individuals. People aged $\geq 65$ years were defined as 'older', in accordance with the definitions used by NHS England. ${ }^{34}$ Other studies that include a wide age range will only be included if outcomes for a $\geq 65$-year-old subgroup are reported.

- Studies: All study designs (including systematic reviews, qualitative research and grey literature) that include older people with advanced CKD, carers/ families in line with the population inclusion criteria outlined including frailty as a descriptor.

- Interventions: Where interventions are the focus of a study, all types of interventions, delivered at any level from individual to service level will be included.

- Outcomes: Health-related outcomes, HRQoL, symptom burden, patent experience, functional and cognitive ability, life participation (this list is not exhaustive and more may be included).

\section{Exclusion criteria}

- Studies not including $\geq 65$-year-old population and studies where subgroup analysis is not possible in this age group.

- Studies not published in English.

- Studies prior to 1995 .

Initially, the search strategy will exclude studies that do not specifically measure frailty or use frailty as a term within the study. This is to provide focus as described above and in the initial searching it became clear this was required to refine the search strategy. Studies are limited to after 1995 to ensure that they are relevant to clinical practice but also as the nosological term frailty is not well reported before 2000. ${ }^{35}$ Studies not published in English will be excluded due to cost and time constraints. This may limit review findings and will be acknowledged in the final summary.

\section{Stage 2: identifying relevant studies} Literature searches

A comprehensive and iterative approach to identify evidence from a range of study designs will be taken. The search will be guided by a health information specialist and incorporate resources that reflect different disciplines while taking a pragmatic approach to meet the required deadlines and resources.

\section{Resources searched}

These are provided in table 1 . Searches will be conducted in three phases: first, scoping (to gauge the volume available and develop/refine the protocol, inclusion and exclusion criteria); second, more comprehensive searches (main literature searches in line with the major resources set out in table 2); and the final stage will be confirming, this involves identification of other sources of information including grey literature and will be decided with further discussion within the core team (searches of reference lists of identified reports/literature, focused website searches, consultation with experts and additional searches following discussions in the sifting phase). The inclusion of grey literature is recommended and will be included to provide a broader view of evidence.

\begin{tabular}{ll}
\hline Table 1 Databases & Focus \\
\hline Database & Biomedical \\
\hline Medline & $\begin{array}{l}\text { Nursing and allied } \\
\text { health }\end{array}$ \\
Cinahl & $\begin{array}{l}\text { Psychology/Mental } \\
\text { health }\end{array}$ \\
\hline Psyclnfo & $\begin{array}{l}\text { Science/Social } \\
\text { sciences }\end{array}$ \\
Web of Science/Web of Knowledge & $\begin{array}{l}\text { General, grey } \\
\text { literature }\end{array}$ \\
\hline Scopus & $\begin{array}{l}\text { Social care, grey } \\
\text { literature }\end{array}$ \\
\hline Social Care Online & Social science \\
\hline $\begin{array}{l}\text { ASSIA } \\
\text { Cochrane Database of Promoting } \\
\text { Health Effectiveness Reviews }\end{array}$ & $\begin{array}{l}\text { Systematic reviews } \\
\text { Embase }\end{array}$ \\
$\begin{array}{l}\text { Renal Association, British Renal } \\
\text { Society and NICE, EDTNA/ERC, } \\
\text { International Nephrology Societies } \\
\text { and affiliated Associations, Academic } \\
\text { organisations }\end{array}$ & $\begin{array}{l}\text { Guidelines and } \\
\text { policies, grey } \\
\text { literature }\end{array}$ \\
\hline & \\
\hline
\end{tabular}




\begin{tabular}{|c|c|c|}
\hline 1 & 2 & 3 \\
\hline $\begin{array}{l}\text { Chronic kidney disease or } \\
\text { CKD (ti,ab) }\end{array}$ & Aged (ti,ab) & Frail (ti,ab) \\
\hline Renal insufficiency (ti,ab) & Aged (exp) & Frailty (exp) \\
\hline Chronic kidney failure (ti,ab) & Geriatric (ti,ab) & $\begin{array}{l}\text { Frail } \\
\text { elderly/frail } \\
\text { (exp) }\end{array}$ \\
\hline Chronic renal failure (ti,ab) & Geriatric (exp) & \\
\hline Chronic kidney disease (ti,ab) & Elderly (ti,ab) & \\
\hline Chronic kidney disease (ti,ab) & Old (ti,ab) & \\
\hline Chronic renal disease (ti,ab) & Old (exp) & \\
\hline
\end{tabular}

Renal failure (ti,ab)

End-stage renal disease (ti,ab)

End-stage kidney disease

(ti,ab)

End-stage renal failure (ti,ab)

End-stage kidney failure (ti,ab)

Established kidney disease

(ti,ab)

Advanced chronic renal

disease or CKD (ti,ab)

End -stage renal disease (ti,ab)

End -stage renal failure (ti,ab)

Renal insufficiency (ti,ab)

Dialysis (ti,ab)

Renal replacement therapy

(ti,ab)

Kidney replacement therapy

(ti,ab)

Renal replacement therapy

(exp)

Renal transplant (ti,ab)

Kidney transplant (ti,ab)

Search looks at any terms in column 1: PLUS any terms in column 2: PLUS any terms in column 3.

CKD, chronic kidney disease; exp, term exploded to incorporate narrower terms; ti,ab, looked in title and abstract.

\section{Search terms (thesaurus and free text)}

This will be identified by scoping searches, an exhaustive list generated by the project team, consulting with the collaborative group and through scoping searches of a small sample of databases. The search will be wide and sensitive to encompass the full range and diversity of perspectives of frail older people with advanced CKD. Search terms used are presented in table 2.

\section{Process of searching}

The searches will be undertaken by a research associate with supervision from an experienced information specialist. Outcomes of the searches will be stored on Endnote web reference management software to enable sharing across the core team and to allow the team to track references throughout the review process. Each search strategy will be recorded to provide a complete history of the search process and to provide transparency of the process.

\section{Stage 3: study selection}

Once the material located in the search stage has been uploaded to Endnote Reference Management Database, core review team members will use the inclusion and exclusion criteria to sift the material. The initial sift will use the study title to identify potentially relevant studies, five members of the team will be involved in this process to ensure consistency. The next sift will be based on title and abstract by two members of the team independently. The third stage will be full article text review, again conducted by two members independently. Documents will be grouped into relevant areas identified by the collaborative group; for example, it may be better to group according to their treatment option, for example, haemodialysis. There is likely to be wide variation and a great deal of heterogeneity in studies involving this particular population. This final third stage (full text sift) will aim to assess the extent, scope and quality of potential evidence; this will be a full-text review to create the final list of included studies. If only abstracts were obtained, they will be included for review. This will be done in duplication, independently by two reviewers to reduce errors and increase validity. We will develop a rapid review assessment tool, based on the inclusion criteria. The core team (five members: $\mathrm{HH}, \mathrm{PO}, \mathrm{AB}, \mathrm{EJ}, \mathrm{ACN}$ and $\mathrm{HY}$ ) will be involved at all stages to strengthen the expertise of the context and methodological considerations. Decisions made will be documented and recorded using Endnote library so a clear decision-making trail is visible. If there is any disagreement regarding potential included studies, the wider collaborative team will be consulted. online supplemental figure 1 represents the stages of the scoping review.

\section{Stage 4: charting the data}

This stage of the review involves extracting key elements of the papers to provide an overview and map of the evidence. These elements have been agreed with the collaborative group and will be recorded in tabular form in Excel spreadsheets under the following suggested headings:

1. Author details and date.

2. Country

3. Aims of the study.

4. Participants and age ranges.

5. Stage of kidney disease and/or dialysis modality.

6. Outcomes mapped to the International Classification for Functioning for Disability and Health Framework (ICF). ${ }^{36}$

7. Geriatric domains studied and frailty assessments used.

8. Study design.

9. Key findings. 
The ICF will provide a recognised framework for reporting the outcomes used, and the interventions described within studies included in this review. The ICF provides a classification of health and health-related domains, including body function and structure, activity and participation. The ICF is the WHO's means of measuring functioning in society irrespective of impairment, and its use will allow gaps in intervention provision and outcome measurement to be readily identified. ${ }^{36}$ The broad categories of impairments of body function and structure, activity and participation will be used to examine outcomes and interventions reported in the studies included within this scoping review and provide a standard structure for reporting findings. ${ }^{37}$ Examples of impairments in body function and structure may include outcomes such as measures of muscle strength, fatigue, mood, balance, cognition, activity (eg, mobility/walking and activities of daily living, such as personal care and meal preparation) and participation (eg, ability to engage in relationships, social roles and community life).

Quality assessments are not typically required in scoping reviews, as scoping studies are not designed to provide a weighting of robust quality evidence or generalisability of results. ${ }^{38}$ A rigorous process will be followed to identify and select studies, but these will not be subject to an in-depth assessment of quality.

\section{Stage 5: collating, summarising and reporting the results}

The scoping review process is iterative by design. It is essential to provide a clear process outlining how the included studies answer the key aims and questions. Initially, the primary analysis will use the tables created in stage 4 to collate and categorise all studies by the agreed criteria. The first tables may be categorised according to prevalence and measures of frailty, or according to RRT modality, and the evidence relating to each presented. The ICF framework will be used to map outcomes relevant to health and disability as described earlier. These tables will then guide the synthesis and be used in the narrative descriptive approach applicable to scoping reviews. ${ }^{33} 38$

Scoping reviews often include stakeholder engagement involving people with lived experience, their carers and family members. ${ }^{38}$ Therefore, the final stage of the review will use focus group discussions to capture patient and carer/family perspectives on the findings of the scoping review. The focus groups will take place within an NHS hospital single centre setting. During the focus group, participants will be presented with the key findings of the review. Nominal group technique (NGT), which is a structured, systematic, transparent and inclusive method for rapidly reaching consensus, will be used to develop consensus. The aim would be to develop research priorities in this population, carer preferences, group and preferred outcome measures. ${ }^{39} 40$ The NGT process will involve asking participants to generate ideas related to the key findings, share and discuss them, and finally vote and rank them in order of priority.
A purposeful approach will be used to identify individuals $>65$ years identified as frail using the Clinical Frailty Scale. Potential participants including family members and carers attending clinics in one centre will be approached. We will also monitor other important characteristics such as gender, ethnicity and stage of CKD/ form of RRT to ensure diverse views are captured. The results of the consensus process will be integrated into the key findings from the scoping review with reference to the review questions and aims.

\section{Data and protection}

The data collected on the participants will be anonymised. The recorded data will be transcribed but remain anonymous with only the participant ID number for the study on all documentation. Other members of the team will have access to the data as part of the analysis but it will remain anonymised.

\section{Patient and public involvement}

Patient representatives have been involved in the design of the scoping review from the outset and embedded as equal partners within the research group. Two key patient leaders have contributed to the writing of the grant proposal and this protocol. Feedback from several kidney patient involvement groups indicated that there was strong support for developing a programme of research to address the specific needs of older people living with frailty and advanced CKD.

\section{Monitoring and governance}

The study will be subject to the audit and monitoring regimen of the Manchester University NHS Foundation Trust (MFT) and follows the MFT Governance protocol.

\section{Ethics and dissemination}

We anticipate publishing these results and continuing the collaboration of the wider interdisciplinary team involved in this review to formulate key, relevant and focused research. This review is funded by the British Renal Society and Kidney Research UK grants committee and findings will be published through their networks and disseminated at national meetings. Two members of the collaborative group are people living with CKD, who will ensure these findings are also published within their national networks. Ethical approval has been granted for the focus group component of this study, and its findings will further elucidate the care and research priorities for this group.

\section{DISCUSSION}

In summary, this review brings together a multiprofessional team of experts to identify and summarise existing evidence relating to relevant research and priorities for older people living with frailty and advanced CKD. Patient involvement in care, shared decisions about their care and increasing advanced care planning have all been cited as important. ${ }^{41-43}$ There is concern that we have a 
growing older population with advanced CKD with an increased prevalence of frailty. Bringing older people with CKD and their carers together and asking them what they think is important to ensure the patient and carer voices are represented.

\section{Indemnity}

This study will be covered by the NHS indemnity scheme.

\section{Author affiliations}

${ }^{1}$ Elderly Health, Manchester University NHS Foundation Trust, Manchester, UK

${ }^{2}$ Renal Dietetics, Manchester University NHS Foundation Trust, Manchester, UK

${ }^{3}$ School of Health and Society, University of Salford School of Nursing Midwifery and Social Work, Manchester, UK

${ }^{4}$ School of Health and Society, University of Salford, Salford, UK

${ }^{5}$ Department of Renal Medicine, Lancashire Teaching Hospitals NHS Foundation

Trust, Preston, UK

${ }^{6}$ Division of Cardiovascular Sciences, The University of Manchester, Manchester, UK ${ }^{7}$ Department of Respiratory Sciences, University of Leicester, Leicester, UK

${ }^{8}$ Leicester Kidney Lifestyle Team, University Hospitals of Leicester NHS Trust, Leicester, UK

${ }^{9}$ Department of Renal Medicine, Leeds Teaching Hospitals NHS Trust, Leeds, UK ${ }^{10}$ Psychology, University of Leeds Leeds Institute of Health Sciences, Leeds, UK

${ }^{11}$ Leeds Institute of Health Sciences, University of Leeds, Leeds, UK

${ }^{12}$ Renal, Imperial College London/Hammersmith Hospital, London, UK

${ }^{13}$ Wolfson Palliative Care Research Centre, Hull York Medical School, University of Hull, Hull, UK

${ }^{14}$ Renal Medicine, Lister Hospital, Stevenage, UK

${ }^{15}$ Kideny Patiemt, Patient Representative, Manchester, UK

Twitter Helen Hurst @hurstnellibert, Andrew Christopher Nixon @andynixons and Hannah Young @hmlyoungphysio

Contributors All authors contributed to study design and obtained study funding. $\mathrm{HH}, \mathrm{PO}, \mathrm{AB}, \mathrm{ACN}, \mathrm{HY}$ and $\mathrm{EJ}$ drafted the protocol and designed the search strategy; these are the core team members. All authors HH, PO, AB, ACN, EJ HY, AM, FEMM, $\mathrm{EB}, \mathrm{DC}, \mathrm{RF}, \mathrm{MDS}-\mathrm{G}, \mathrm{AW}$ and HB have read and approved the final protocol, this is the whole collaborative team.

Funding This work was supported by British Renal Society and Kidney Care UK grant joint partnership. HY is supported by a grant from the NIHR (DRF-2016-09015). The views expressed in this publication are those of the authors and not necessarily those of the NHS, the National Institute for Health Research or the Department of Health and Social Care. The funders have no role in the study design; collection, analysis and interpretation of the data; writing the report; and the decision to submit the report for publication.

Competing interests None declared.

Patient consent for publication Not required.

Provenance and peer review Not commissioned; externally peer reviewed.

Supplemental material This content has been supplied by the author(s). It has not been vetted by BMJ Publishing Group Limited (BMJ) and may not have been peer-reviewed. Any opinions or recommendations discussed are solely those of the author(s) and are not endorsed by BMJ. BMJ disclaims all liability and responsibility arising from any reliance placed on the content. Where the content includes any translated material, BMJ does not warrant the accuracy and reliability of the translations (including but not limited to local regulations, clinical guidelines, terminology, drug names and drug dosages), and is not responsible for any error and/or omissions arising from translation and adaptation or otherwise.

Open access This is an open access article distributed in accordance with the Creative Commons Attribution Non Commercial (CC BY-NC 4.0) license, which permits others to distribute, remix, adapt, build upon this work non-commercially, and license their derivative works on different terms, provided the original work is properly cited, appropriate credit is given, any changes made indicated, and the use is non-commercial. See: http://creativecommons.org/licenses/by-nc/4.0/.

\section{ORCID iDs}

Helen Hurst http://orcid.org/0000-0002-7268-171X

Andrew Christopher Nixon http://orcid.org/0000-0002-4093-6204
REFERENCES

1 Digital N. Hospital admissions patient care activity 2015-2016 NHS digital. London, 2016.

2 Clegg A, Young J, Iliffe S, et al. Frailty in elderly people. The Lancet 2013;381:752-62.

3 Fried LP, Tangen CM, Walston J, et al. Frailty in older adults: evidence for a phenotype. J Gerontol A Biol Sci Med Sci 2001;56:M146-56.

4 Mitnitski AB, Mogilner AJ, Rockwood K. Accumulation of deficits as a proxy measure of aging. ScientificWorldJournal 2001;1:323-36.

5 BGS. Fit for frailty. British geriatric Society, 2014. Available: http:// www.bgs.org.uk/campaigns/fff/fff_full.pdf

6 Rockwood K, Song X, MacKnight C, et al. A global clinical measure of fitness and frailty in elderly people. CMAJ 2005;173:489-95.

7 Walston JD, Bandeen-Roche K. Frailty: a tale of two concepts. BMC Med 2015;13:185.

8 Clegg A, Rogers L, Young J. Diagnostic test accuracy of simple instruments for identifying frailty in community-dwelling older people: a systematic review. Age Ageing 2015;44:148-52.

9 Walston J, Robinson TN, Zieman S, et al. Integrating frailty research into the medical Specialties-Report from a U13 conference. J Am Geriatr Soc 2017;65:2134-9.

10 McAdams-DeMarco MA, Law A, Salter ML, et al. Frailty as a novel predictor of mortality and hospitalization in individuals of all ages undergoing hemodialysis. J Am Geriatr Soc 2013;61:896-901.

11 Chowdhury R, Peel NM, Krosch M, et al. Frailty and chronic kidney disease: a systematic review. Arch Gerontol Geriatr 2017;68:135-42.

12 Johansen KL, Chertow GM, Jin C, et al. Significance of frailty among dialysis patients. J Am Soc Nephrol 2007;18:2960-7.

13 Roshanravan B, Khatri M, Robinson-Cohen C, et al. A prospective study of frailty in nephrology-referred patients with CKD. Am J Kidney Dis 2012;60:912-21.

14 Bao Y, Dalrymple L, Chertow GM, et al. Frailty, dialysis initiation, and mortality in end-stage renal disease. Arch Intern Med 2012;172:1071-7.

15 McAdams-DeMarco MA, Suresh S, Law A, et al. Frailty and falls among adult patients undergoing chronic hemodialysis: a prospective cohort study. BMC Nephrol 2013;14:224.

16 McAdams-DeMarco MA, Tan J, Salter ML, et al. Frailty and cognitive function in incident hemodialysis patients. Clin J Am Soc Nephrol 2015;10:2181-9.

17 Mansur HN, Colugnati FA, Grincenkov FRDS, et al. Frailty and quality of life: a cross-sectional study of Brazilian patients with pre-dialysis chronic kidney disease. Health Qual Life Outcomes 2014;12:27.

18 Lee SJ, Son H, Shin SK. Influence of frailty on health-related quality of life in pre-dialysis patients with chronic kidney disease in Korea: a cross-sectional study. Health Qual Life Outcomes 2015;13:70.

19 lyasere OU, Brown EA, Johansson L, et al. Quality of life and physical function in older patients on dialysis: a comparison of assisted peritoneal dialysis with hemodialysis. Clin J Am Soc Nephrol 2016;11:423-30.

20 Nixon AC, Bampouras TM, Pendleton N, et al. Frailty is independently associated with worse health-related quality of life in chronic kidney disease: a secondary analysis of the frailty assessment in chronic kidney disease study. Clin Kidney J 2019.

21 Zhao Y, Liu Q, Ji J. The prevalence of frailty in patients on hemodialysis: a systematic review and meta-analysis. Int Uro Nephrol 2020;52:115-20.

22 Tong A, Manns B, Hemmelgarn B, et al. Establishing core outcome domains in hemodialysis: report of the standardized outcomes in Nephrology-Hemodialysis (SONG-HD) consensus workshop. Am J Kidney Dis 2017;69:97-107.

23 Morton RL, Devitt J, Howard K, et al. Patient views about treatment of stage 5 CKD: a qualitative analysis of semistructured interviews. Am J Kidney Dis 2010;55:431-40.

24 Tong A, Cheung KL, Nair SS, et al. Thematic synthesis of qualitative studies on patient and caregiver perspectives on end-of-life care in CKD. Am J Kidney Dis 2014;63:913-27.

25 Akpan A, Roberts C, Bandeen-Roche K, et al. Standard set of health outcome measures for older persons. BMC Geriatr 2018;18:36.

26 Alliance JL. Frailty Canada top 10, 2018. Available: http:// wwwjlanihracuk/priority-setting-partnerships/frailty-canada/top-10prioritieshtm

27 Garrard JW, Cox NJ, Dodds RM, et al. Comprehensive geriatric assessment in primary care: a systematic review. Aging Clin Exp Res 2020;32:197-205.

28 van Loon IN, Wouters TR, Boereboom FTJ, et al. The relevance of geriatric impairments in patients starting dialysis: a systematic review. Clin J Am Soc Nephrol 2016;11:1245-59.

29 Farrington K, Covic A, Nistor I, et al. Clinical Practice Guideline on management of older patients with chronic kidney disease stage $3 \mathrm{~b}$ 
or higher (eGFR $<45 \mathrm{~mL} / \mathrm{min} / 1.73 \mathrm{~m} 2)$ : a summary document from the European Renal Best Practice Group. Nephrol Dial Transplant 2017;32:9-16.

30 Parker SG, McCue P, Phelps K, et al. What is comprehensive geriatric assessment (CgA)? an umbrella review. Age Ageing 2018;47:149-55.

31 Hannah MI. Frailty and falls: a qualitative study exploring the experiences of patients living with end-stage renal disease and haemodialysis. World Congress of Physio 2019 UK 2018.

32 Tricco AC, Lillie E, Zarin W, et al. PRISMA extension for scoping reviews (PRISMA-ScR): checklist and explanation. Ann Intern Med 2018;169:467-73.

33 Levac D, Colquhoun H, O'Brien KK. Scoping studies: advancing the methodology. Implement Sci 2010;5:69.

34 . Available: https://www.england.nhs.uk/ourwork/clinical-policy/ older-people/improving-care-for-older-people

35 Pickard S. Frail bodies: geriatric medicine and the constitution of the fourth age. Sociol Health IIIn 2014;36:549-63.

36 The International classification for functioning for disability and health (ICF). Available: https://wwwwhoint/classifications/icf/en/
37 World Health O. International classification of functioning, disability and health : ICF. Geneva: World Health Organization, 2001.

38 Arksey H, O'Malley L. Scoping studies: towards a methodological framework. Int J Soc Res Methodol 2005;8:19-32.

39 McMillan SS, Kelly F, Sav A, et al. Using the nominal group technique: how to analyse across multiple groups. Health Serv Outcomes Res Method 2014;14:92-108.

40 Young H, Goodliffe S, Madhani M, et al. Co-producing progression criteria for feasibility studies: a partnership between patient contributors, clinicians and researchers. Int J Environ Res Public Health 2019;16:3756.

41 Winterbottom A, Bekker H, Mooney A. Dialysis modality selection: physician guided or patient led? Clin Kidney J 2016;9:823-5.

42 Davison SN, Jhangri GS. Existential and supportive care needs among patients with chronic kidney disease. J Pain Symptom Manage 2010;40:838-43.

43 Davison S, Simpson C. Facilitated advance care planning in end stage renal disease enhanced patients ' hope. BMJ 2006;333:1-5. 